\title{
Isolation and characterization of the active cDNA of the human cell cycle gene (RCC1) involved in the regulation of onset of chromosome condensation
}

\author{
Motoaki Ohtsubo, ${ }^{1}$ Ryosuke Kai, ${ }^{1,4}$ Nobuaki Furuno, ${ }^{1}$ Takeshi Sekiguchi, ${ }^{1,5}$ Mutsuo Sekiguchi, ${ }^{1,6}$ \\ Hidenori Hayashida, ${ }^{2}$ Kei-ichi Kuma, ${ }^{2}$ Takashi Miyata, ${ }^{2}$ Shinichi Fukushige, ${ }^{3}$ Tomoaki Murotsu, ${ }^{3}$ \\ Kenichi Matsubara, ${ }^{3}$ and Takeharu Nishimoto ${ }^{1,5}$
}

The human RCC1 gene was cloned after DNA-mediated gene transfer into the tsBN2 cell line, which shows premature chromosome condensation at nonpermissive temperatures $\left(39.5-40^{\circ} \mathrm{C}\right)$. This gene codes for a $2.5-\mathrm{kb}$ poly $(A)^{+}$RNA that is well conserved in hamsters and humans. We isolated 15 cDNA clones from the Okayama-Berg human cDNA library, and found two that can complement the tsBN2 mutation with an efficiency comparable to that of the genomic DNA clone. The base sequences of these two active cDNA clones differ at the $5^{\prime}$ proximal end, yet both have a common open reading frame, encoding a protein of $\mathbf{4 2 1}$ amino acids with a calculated molecular weight of 44,847 and with seven homologous repeated domains of about 60 amino acids. This human RCC1 gene was located to human chromosome 1 using sorted chromosomal fractions.

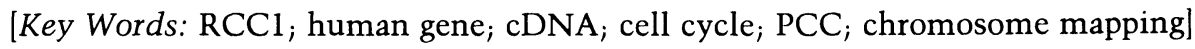

Received April 28, 1987; revised version accepted June 11, 1987.

Mitotic cells possess the chromosome-condensing factor(s), the presence of which is shown by fusing mitotic and interphase cells (Johnson and Rao 1970). Upon fusion, the chromatin is condensed and exhibits various forms of prematurely condensed chromosomes (PCCs), depending on the cell cycle phase (Johnson and Rao 1970; Rao et al. 1976). In mitotically condensed chromosomes, DNA is packed between 5000- and 10,000-fold. Except for small-sized RNA, RNA cannot be transcribed from such packed DNA (Prescott 1976). Following condensation of the chromatin, the nuclear membrane is broken down and the synthesis of protein is repressed. If the chromosome condensation occurs in the S phase, DNA replication ceases simultaneously (Nishimoto et al. 1978, 1981). Thus, while the condensation of chromatin is risky for cell survival, it is essential for the even distribution of genetic material into daughter cells (Prescott 1976). Therefore, to carry out the condensation process safely and reversibly, cells must have regulatory mechanisms that will allow condensation of the chro-

${ }^{1}$ Laboratory of Molecular Genetics, ${ }^{2}$ Laboratory of Theoretical Biology, Department of Biology, Faculty of Science, Kyushu University 33, Fukuoka 812, Japan.

${ }^{3}$ Institute for Molecular and Cellular Biology, Osaka University, Yamada-oka, Suita 565, Japan.

${ }^{4}$ Deceased (May 22, 1986, at age 29).

${ }^{5}$ Present address: Department of Molecular Biology, Graduate School of Medical Science, Kyushu University, Fukuoka 812, Japan.

6Present address: Department of Biochemistry, Faculty of Medicine, Kyushu University, Fukuoka 812, Japan. matin to occur at a precise time in the cell cycle. In the normal cell cycle, the chromosome-condensing factor(s) appears in the early $G_{2}$ phase and accumulates toward the mitotic phase (Sunkara et al. 1979). If this regulatory mechanism is defective, the chromatin will be irregularly condensed, causing cell death. Thus, mutations associated with this step in the cell cycle have to be isolated as temperature-sensitive $(t s)$ mutants.

The $t s \mathrm{BN} 2$ cell line derived from the BHK21/13 cell line seems to be one such mutant. At the nonpermissive temperature, $t s \mathrm{BN} 2$ cells show premature chromosome condensation (Nishimoto et al. 1978, 1981). This phenomenon can be observed with a microscope during the period from $S$ phase to $G_{2}$ phase. The typical PCC figures of the $S$ and $G_{2}$ phases observed upon cell fusion with mitotic cells are induced by switching the permissive temperature to the nonpermissive one. In addition to PCC, other mitosis-specific phenomena, such as the mitosis-specific phosphorylation of histone and nonhistone proteins, are induced simultaneously with PCC (Ajiro et al. 1983; Yamashita et al. 1985). Furthermore, $t s B N 2$ cells showing PCC can condense interphase chromatin upon cell fusion. Thus, the chromosome-condensing factor is newly produced in tsBN2 cells at the nonpermissive temperature (Hayashi et al. 1982). This is compatible with the finding that cycloheximide, a potent inhibitor of protein synthesis, prohibits the induction of PCC in tsBN2 cells (Nishimoto et al. 1981). These observations suggest that the mechanism regulating the 
Ohtsubo et al.

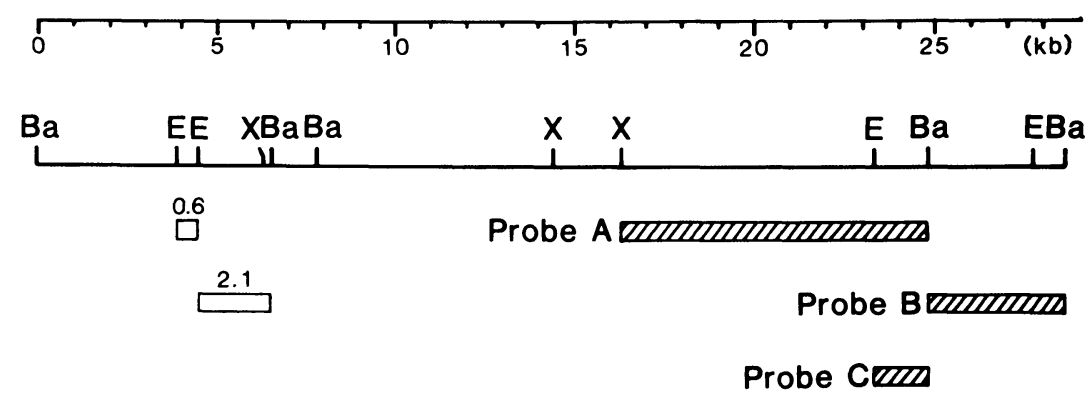

Figure 1. Restriction map of the human $\mathrm{RCCl}$ gene. The human DNA region shared by cosmids B95 and B4 is described. The positions of probes used for RNA analysis and cDNA cloning are shown by cross-hatched bars. The positions of the 0.6-kb E-E fragment and the 2.1-kb E-Ba fragment are indicated by rectangles. Restriction endonuclease sites: $(\mathrm{Ba})$ BamHI; (E) EcoRI; (X) XhoI.

production of chromosome-condensing protein is temperature-sensitive in $t s \mathrm{BN} 2$ cells.

To investigate mutation at the molecular level, the best method is to clone the gene that can complement the defective character of mutants. In case of animal cell lines, several human genes, including the human repair gene ERCCl, have been cloned by DNA-mediated gene transfer (Marcel et al. 1986). Although such cloned genes are not absolutely defective in the mutant, and may even be a suppressor gene, these cloned genes are no doubt involved in the defective pathway of a mutant and so can complement the mutation.

We cloned a human gene, designated $\mathrm{RCCl}$, which can transform $t s B N 2$ cells to the ts $^{+}$phenotype and which has a size of about $30 \mathrm{kbp}$ (Kai et al. 1986). We now report the cDNA cloning and chromosomal mapping of this RCCl gene. The cDNA of the RCCl gene encodes a protein of 421 amino acids with seven repeated sequences. The RCCl gene is located on chromosome 1 .

\section{Results}

\section{Cloning of RCC1 cDNA}

The human RCCl gene was cloned into two cosmid DNAs, B95 and B4, from the secondary ts $^{+}$transformant of $t s \mathrm{BN} 2$ cells, ST2-7. The two cosmid DNAs can transform $t s B N 2$ cells to the ts ${ }^{+}$phenotype, with an efficiency exceeding 1000-fold, compared with transfection with total human DNA (Kai et al. 1986). The restriction map of the human DNA region shared by two cosmids is shown in Figure 1.

To isolate the cDNA of the RCCl gene, the putative human RCC1 DNA was fragmented and subcloned into pBR322, probes A and B (Fig. 1). Although probes A and B hybridized with a $2.5-\mathrm{kb}$ RNA, these probes contain a lot of the human repeated sequence. Probe $\mathrm{C}$ was found to be repeat-free and was used to screen the human expression cDNA library made from a SV40-transformed human fibroblast generously provided by Dr. P. Berg (Okayama and Berg 1983). A total of $8.5 \times 10^{5}$ colonies were screened and 15 positive clones were isolated. Three (pcD32, pcD40, and pcD51) were characterized.

Northern blot analysis of the poly $(\mathrm{A})^{+}$RNA of HeLa cells revealed that the cDNA clone hybridized mainly to a poly $(\mathrm{A})^{+}$RNA of $2.5 \mathrm{~kb}$. The 2.5 -kb RNA species is also observed in $t s \mathrm{BN} 2$ cells, as is the secondary ts ${ }^{+}$ transformant, ST2-7 (Fig. 2). Thus, the $2.5-\mathrm{kb}$ poly $(\mathrm{A})^{+}$ RNA, which is well conserved between hamsters and humans, is a candidate for the human $\mathrm{RCCl}$ transcript. In $t s \mathrm{BN} 2$ cells that have been cultured at the permissive

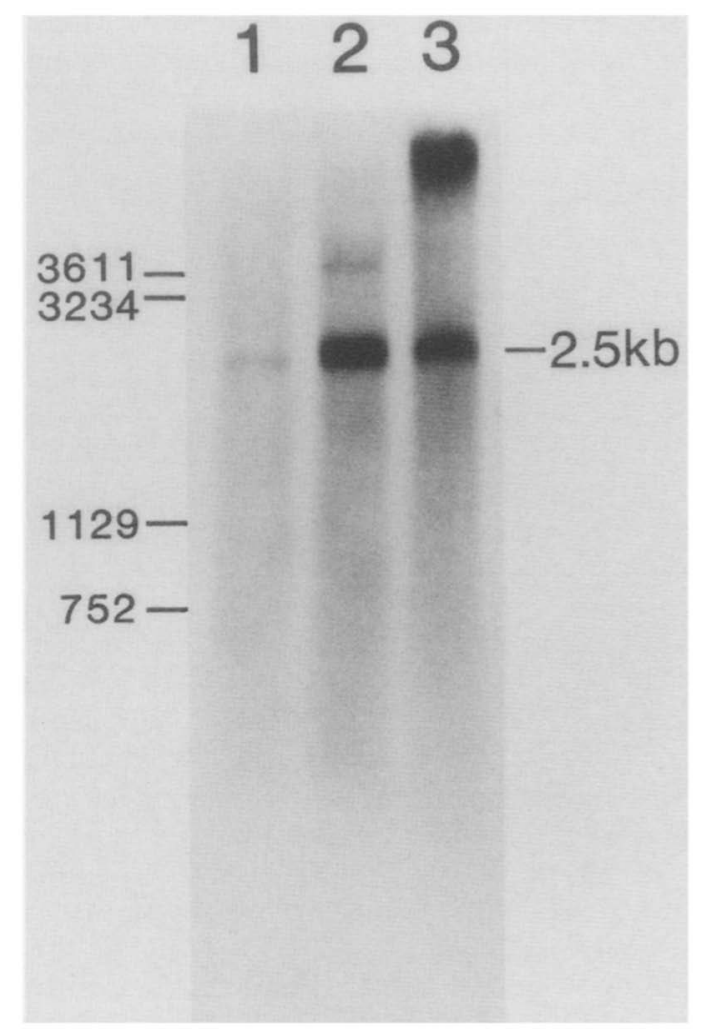

Figure 2. Northern blot analysis of poly $(\mathrm{A}))^{+} \mathrm{RNA}$ from HeLa cells. Cells were cultured at $33.5^{\circ} \mathrm{C}(t s \mathrm{BN} 2$ cells $), 37.5^{\circ} \mathrm{C}(\mathrm{HeLa}$ cells), and $39.5^{\circ} \mathrm{C}$ (ST2-7 cells). Poly(A) ${ }^{+}$RNAs extracted from the cytoplasmic RNA of $t s B N 2$ (lane 1) and ST2-7 (lane 2) cells and from the total cellular RNA of HeLa (lane 3) cells were electrophoresed in a $1.5 \%$ agarose gel containing formaldehyde and, after transfer to nitrocellulose, were hybridized to a $32 \mathrm{P}-\mathrm{la}$ beled RCCl cDNA. The amount of applied poly(A) ${ }^{+}$RNA is as follows: (lane 1) tsBN2 $(10 \mu \mathrm{g})$; (lane 2) ST2-7 (12.6 $\mu \mathrm{g})$; and (lane 3) HeLa $(9.4 \mu \mathrm{g})$. The size of RNA was estimated by coelectrophoresis of mixed pBR322 DNAs digested by PstI and EcoRI or by PstI and BamHI. 


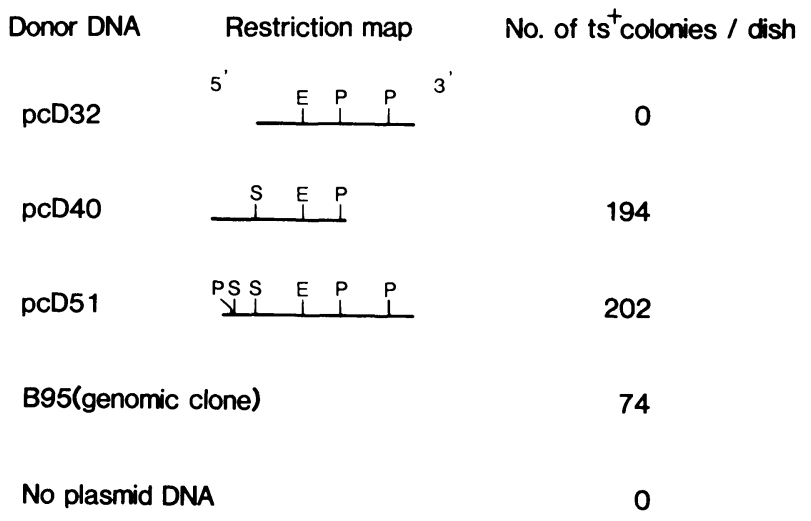

Figure 3. Restriction map and the biological activity of isolated $\mathrm{RCCl} \mathrm{cDNA}$ clones. The restriction maps of $\mathrm{RCCl}$ cDNA clones pcD32, pcD40, and pcD51 were determined using the restriction enzymes PstI (P), EcoRI (E), and SphI (S). Lengths of the pcD RCCl clones are as follows: pcD32, $2.0 \mathrm{~kb}$; pcD40, $1.7 \mathrm{~kb}$; pcD51, $2.4 \mathrm{~kb}$. tsBN2-N9 cells $\left(7.5 \times 10^{5}\right.$ cells $)$ were plated into a $100-\mathrm{mm}$ dish and transfected with $1 \mu \mathrm{g}$ of $\mathrm{pcD}$ DNA or $0.4 \mu \mathrm{g}$ of cosmid B95 DNA and, as a carrier, with $20 \mu \mathrm{g}$ of $t s \mathrm{BN} 2 \mathrm{DNA}$. Transfected cells were incubated at $39.5^{\circ} \mathrm{C}$ for 9 days and the number of $\mathrm{ts}^{+}$colonies counted.

temperature, this RNA is somewhat smaller than that in HeLa cells. The poly $(\mathrm{A})^{+}$RNA of $t s \mathrm{BN} 2$ and ST2-7 cells was fractionated from cytoplasmic RNA; thus, the $\sim 4-\mathrm{kb}$ band of RNA observed only in ST2-7 cells may not be a precursor RNA of the human RCCl mRNA. This 4-kb RNA probably was transcribed from the rearranged human $\mathrm{RCCl}$ gene transfected into ST2-7 cells, because in ST2-7 cells the transfected human DNA was amplified and several rearranged human DNA clones were isolated from the genomic DNA library of ST2-7 cells (data not shown). The poly(A) ${ }^{+}$RNA of HeLa cells was derived from the total cellular RNA, which was extracted with the hot phenol method (Maniatis et al. 1982). Because this RNA extraction method cannot prevent DNA from contaminating the RNA fraction, the $\sim 10-\mathrm{kb}$ band in HeLa cells (Fig. 2, lane 3) may be the contaminated nuclear DNA.

The aligned restriction maps of the three representative cDNA clones are shown in Figure 3. Two of them, pcD40 and pcD51, can transform tsBN2 cells to the ts ${ }^{+}$ phenotype. The pcD32 clone, which has the same 3' portion as the pcD51 clone, cannot complement the $t s \mathrm{BN} 2$ mutation; thus, the $5^{\prime}$ portion defective in pcD32 is necessary for expression of biological activity. The pcD40 and pcD51 clones complemented the tsBN2 mutation with the same efficiency as observed with the genomic clone B95. Since the DNA in pcD clones is expressed under control of the SV40 promoter, which has no signal for cell-cycle-specific transcription (Okayama and Berg 1983), this signal may not be required for complementing the $t s \mathrm{BN} 2$ mutation.

The restriction maps in the $5^{\prime}$ region of the two active cDNA clones differ (Figs. 3 and 5, below). Sequence analysis of the two clones (shown below) revealed that the 5 '-proximal end of pcD40 differs from that of pcD51. This was confirmed by Southern blot analysis (Fig. 4).
The cosmid B95 DNA was completely digested with $B a m H I$ or with two restriction enzymes, BamHI and EcoRI, and hybridized to the 5' part of EcoRI fragments of the pcD40 and pcD51 clones, according to Southern analysis (Southern 1975). Both cDNA fragments were hybridized with the same two BamHI fragments 16.6 and $17 \mathrm{~kb})$. However, after digestion with EcoRI and BamHI, pcD40 was hybridized to the $0.6-\mathrm{kb}$ band, in addition to the $17-\mathrm{kb}$ band, and pcD51 was hybridized to the $2.1-\mathrm{kb}$ band, in addition to the $17-\mathrm{kb}$ band. The $0.6-\mathrm{kb}$ and 2.1 $\mathrm{kb}$ bands are derived from the $6.6 \mathrm{~kb}$ BamHI fragment located at the $5^{\prime}$-proximal region of the human $\mathrm{RCCl}$ gene (Fig. 1). Thus, two pcD40 and pcD51 cDNAs seem to be transcribed from a different promoter in the same human $\mathrm{RCCl}$ gene.

Both active cDNA clones have a common open reading frame. However, the $3^{\prime}$ untranslated region of $\mathrm{pcD} 40$ is shorter than that of pcD51. Sizes of the isolated cDNA varied from $1.7 \mathrm{kbp}$ to $2.4 \mathrm{kbp}$, the latter corresponding to the size of the mRNA $(2.5-2.6 \mathrm{~kb})$. Hence, we presume that the entire RCCl cDNA was isolated.

\section{Sequence of RCC1 $c D N A$}

Following the strategy depicted in Figure 5, the nucleotide sequences of the cDNA clones pcD40 and pcD51

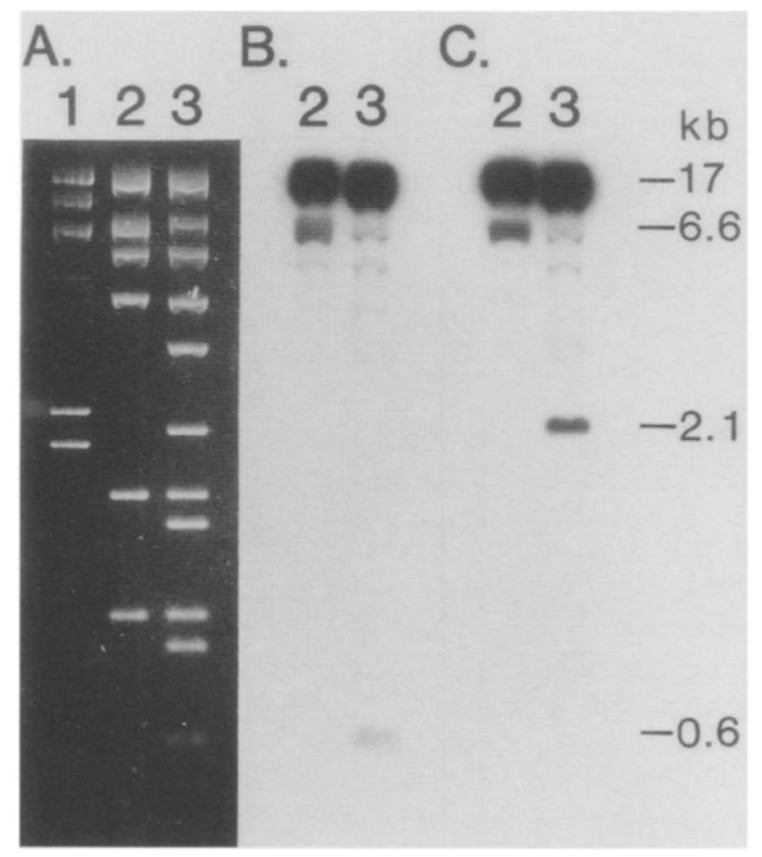

Figure 4. Southern blot analysis of the $5^{\prime}$ coding region in the human RCCl gene. The cosmid B95 DNA was completely digested with BamHI or BamHI and EcoRI, and then applied to a $1.0 \%$ horizontal agarose gel. After electrophoresis, DNAs were stained, photographed, and blotted onto nitrocellulose papers. The paper was hybridized to the nick-translated, ${ }^{32} \mathrm{P}$-labeled $5^{\prime}$ EcoRI fragment of pcD40 $(B)$ or pcD51 $(C)$. The positions of molecular weights were determined by co-electrophoresis of HindIII-digested $\lambda$ cI857 DNA. (A) Photograph of the ethidium bromide-stained gel. $(B$ and $C) \mathrm{X}$-ray film of the hybridized blot. Lanes: (1) $\lambda c I 857$ DNA digested with HindIII; (2) BamHI digested B95 DNA; (3) B95 DNA digested with BamHI and EcoRI. 


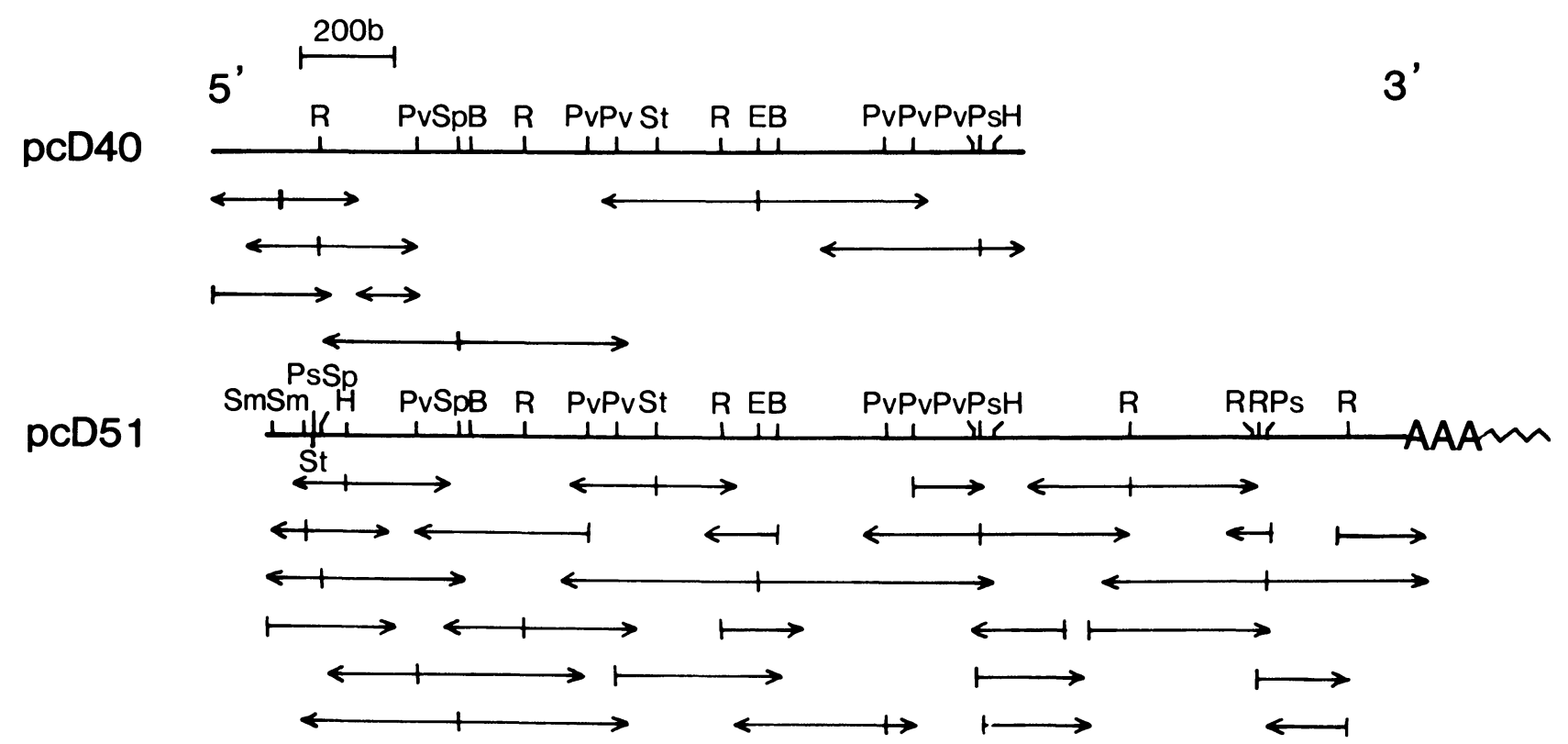

Figure 5. Sequence strategy of the active RCCl clones pcD40 and pcD51. Fine restriction cleavage maps of two active RCC1 cDNA clones were determined using the following restriction enzymes: RsaI (R), PvuII (Pv), SphI (Sp), BalI (B), StuI (St), EcoRI (E), PstI (Ps), $\operatorname{SmaI}(\mathrm{Sm})$, and HgiAI (H).

were determined by the dideoxy nucleotide method (Sanger et al. 1977). The nucleotide sequences and deduced amino acid sequences of two active cDNA clones are shown in Figure 6.

The pcD40 clone contains a 1724-bp insert with a 1263-bp open reading frame (ORF) and the pcD51 clone contains a 2424-bp insert with a 1425-bp ORF. Both cDNAs share the 1436-bp region in which a 1263 -bp region constitutes an ORF. In pcD40, this 1263-bp ORF is preceded by a region of 320 bp containing two in-frame termination codons, indicating that this ORF is the only large open reading frame in pcD40. Since both cDNAs possess much the same activity for complementing the tsBN2 mutation, this 1263-bp ORF shared by both cDNAs may encode the human RCCl protein. This argument is further confirmed by the following evidence. The putative start codon of the RCCl protein has the purine residue adenine at the -3 position, which has been found in most eukaryotic ATG start codons (Kozak 1984). Thus, we conclude that the human RCCl cDNA encodes a protein of 421 amino acids with a calculated molecular weight of 44,847 .

The pcD40 clone does not have the common polyadenylation signal AATAAA (Proudfoot and Brownlee 1976) in the $3^{\prime}$ untranslated region, but pcD51 has this AATAAA signal at 13 bases upstream of the poly(A) tail. In pcD51, another AATAAA is present 773 bases upstream of the poly(A) tail, which is 38 bases downstream from the poly(A) additional site of pcD40. The pcD40 clone has, at 49 and 93 bases upstream of the poly(A) tail, the CAYTG sequences found adjacent to the common polyadenylation site in many eukaryotic mRNAs (Berget 1984).

\section{Tandem repetition within the putative $R C C 1$ protein}

Structural analysis of the putative human $\mathrm{RCCl}$ product was done using a computer program for homology searching, as described in Toh et al. (1983). A comparison of the amino acid sequence predicted from the cDNA with itself revealed a unique pattern of homology indicating the presence of seven tandem repeats of about 60 residues (Fig. 7). On the basis of the homology matrix shown in this figure, the amino acid sequences of the homologous units were aligned (Fig. 8). The seven repeats share identical or chemically similar amino acids at several positions (marked by circles in Fig. 8), suggesting strongly that the observed homologies have a biological significance. Interestingly, the glycine content is rich in these repeats and the glycines are strongly conserved at four positions among the different repeats. These conserved residues may play an important role in structure and/or function of the human $\mathrm{RCCl}$ product. Comparison of the amino acid sequence of the RCC1 product with the published sequence compiled in the National Biomedical Research Foundation (NBRF) protein data base (1986) revealed no significant homology.

\section{Chromosomal localization of RCC1 gene}

Metaphase chromosomes of the human lymphoblastoid cell line GM0131 were sorted into eight chromosome fractions $(A-H$, Fig. 9) using a fluorescence-activated cell sorter. DNA was extracted from each chromosome fraction, digested with PvulI, and subjected to Southern hybridization tests (Southern 1975), using the DNA fragment $C$ in Figure 1 as a probe. As shown in Figure 9, fraction A, along with the total DNA of GM0131 (lane L, 
cDNA and chromosomal location of RCC1

\begin{abstract}
A pcD40

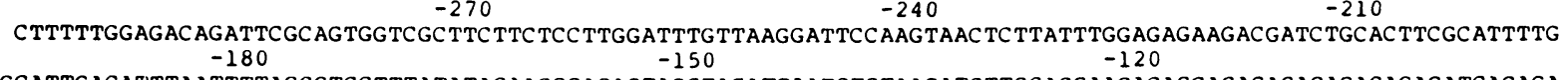

GCATTGACATTTAATTTTAGG TCCTTTATATAGAAGGGAGAGTAGCTACATGAATGTGTAAGATCTTGGAGGAAGACAGCAGAGAGAGAGAGAGAGATCAGAGA

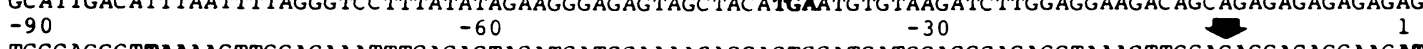
TCCCAGGGTTAAAAGTTGGAGAATTTCACAGTACATCATCCAAAAGAGGAGTCCATGATGGAGGCAGAGG TAAAC TTGGAGAGGACAGGAAGATGTCACCCAAG 30
CGCATAGCTAAAAGAAGGTCCCCCCAGCAGATGCCATCCCCAAAAGCAAGAAGG TGAAGGTCTCACACAGGTCCCACAGCACAGAACCCGGCTTGGTGCTGACA

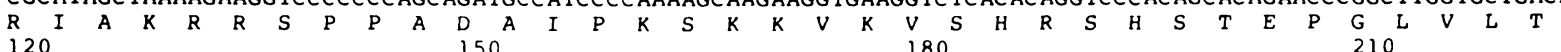

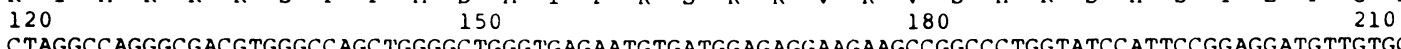
CTAGGCCAGGGCGACGTGGGCCAGCT'GGGGCTGGGTGAGAATGTGATGGAGAGGAAGAAGCCGGCCCTGGTATCCATTCCGGAGGATGTTGTGCAGGCTGAGGCT

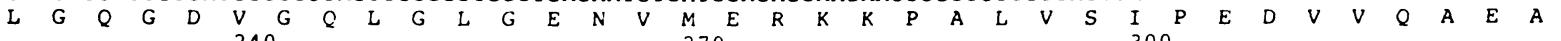
GGGGGCATGCACACCGTGTGTCTAAGCAAAAGTGGCCAGGTCTATTCCTTCGGCTGCAATGATGAGGGTGCCCTGGGAAGGGACACATCAGTGGAGGGCTCGGAG

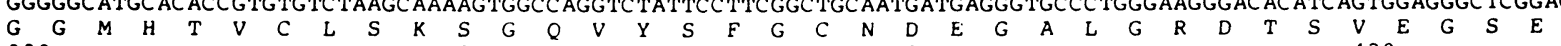
$33003600390 \quad 420$ ATGGTCCCTGGGAAAG TGGAGC TGCAAGAGAAGGTGGTACAGGTGTC AGC AGGAGACAGTCACACAGCAGCCC TCACCGATGATGGCCGTGTCTTCCTC TGGGGC $\begin{array}{lllllllllllllllllllllllllllllllllllll}M & V & P & G & K & V & E & L & G & E & K & V & V & Q & V & S & A & G & D & S & H & T & A & A & L & T & D & D & G & R & V & F & L & W & G\end{array}$ TCCTTCCGGGACAATAACGGTGTGATTGGACTGTTGGAGCCCATGAAGAAGAGCATGGTGCCTGTGCAGGTGCAGCTGGATGTGCC TGTGGTAAAGGTGGCCTCA

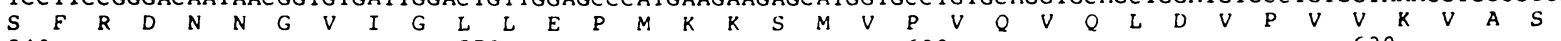
540 ( 570 2000 GGAAACGACCACTTGGTGATGCTGACAGCTGATGGTGACCTCTACACCTTGGGCTGCGGGGACAGGGCCAGC TAGGCCGTGTGCCTGAGTTATTTGCCAACCGT

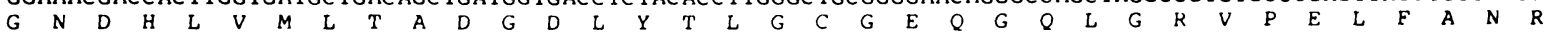

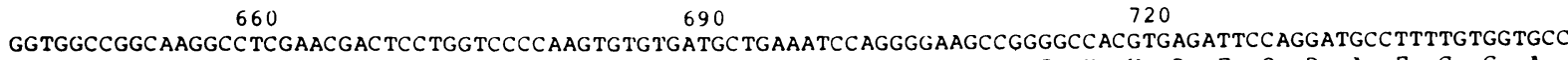

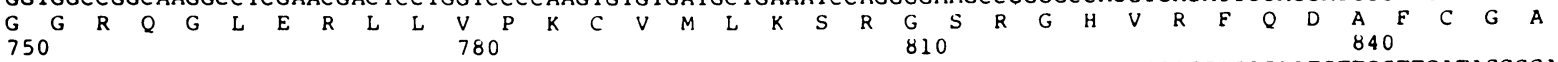
TATTTCACCTTTGCCATCTCCCATGAGGGCCACGTGTACGGCTTCGGCC TC TCCAACTACCATCAGCTTGGAACTCCGGGCACAGAATC TTGC TTCATACCCCAG

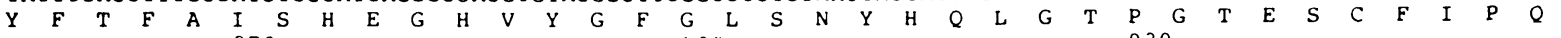

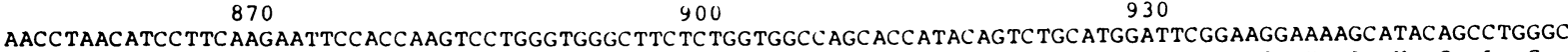

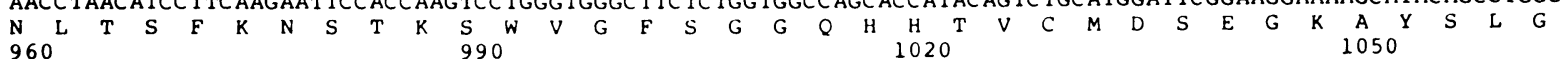

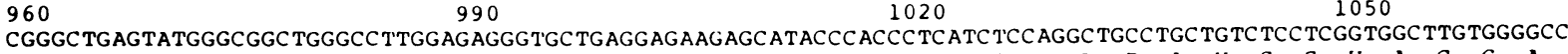
$\begin{array}{llllllllllllllllllllllllllllllllllll}R & A & E & Y & G & R & L & G & L & G & E & G & A & E & E & K & S & I & P & T & L & I & S & R & L & P & A & V & S & S & V & A & C & G & A\end{array}$ $R$ A E $Y$ G $R$ R $L$ G L G E G A E E 1110 S TCTGTGGGGTATGCTGTGACCAAGGATGGTCGTGTTTTCGCCTGGGGCATGGGCACCAAC TACCAGC TGGGCACAGGGCAGGATGAGGACGCCTGGAGCCCTGTG

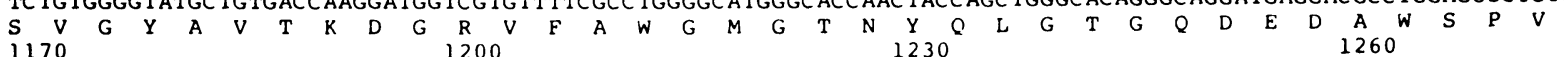

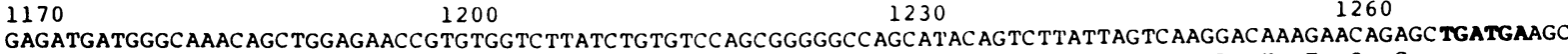

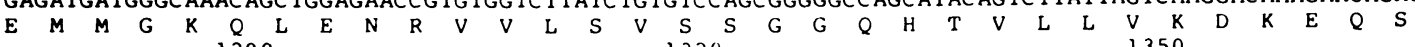

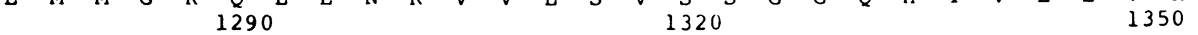
CTCTGAGGGCCTGGC TTC TGTCCTGCACAACCTCCCTCACAGAACAGGGAAGCAGTGACAGCTGCAGATGGCAGCGGGCCTC TCCCCAGCCCTGAGCACTGTGTC 1380 AGTTCCTGCCTTTTCTCATCAGCAGAACAGAATCCTTTTCCTCTTAAAAAAAAAAAAAAAAA -.....

8 pcD51

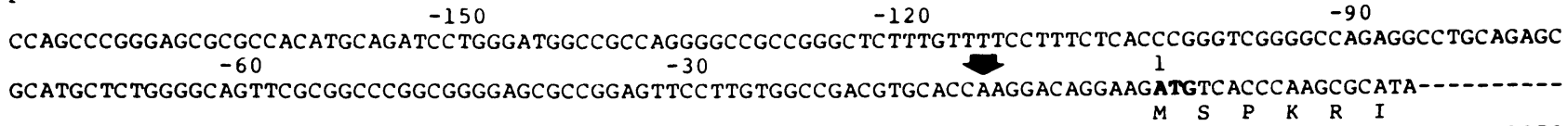
1272 S

AGTTCCTGCCTTTTCTCATCAGCAGAACAGAATCCTTTTCCTCTTTTCCTTCCTCCTCTTTGGAATTTTCCTGGGACCTACAGAATAMAGGGGGGATGGACAGG GGGTTTTCAAAAGGAACATGGCTCACTCAGAGCTATATGGTTAGACGTTTC TCCCCTTTTCCCTACCTTCCATGGTCCTGGTTGGCCCTGGCTTTGCCTACTAGA AAACCAAAACTTCCCCCCTGGGGTTTTGTGCCCACTCTCTGAGAAGTTGGGGCTCCATCAAGCCCCATTCTAGTCATGTGCCCCTTTCCTGTCCCTAACAGTCCA CAGGCAAACAAATGGTACAGTCATAAGAGCCATCTGTCACGGACCCACGCCCAGAGGAACGTGCAGAAAAAAGCAGAGCTACATGGCTGTGGGCAACTATAAGCC AAATATTTGGCTCAGAACAGGTGTCCATGGGACAAAAAAGAACGATCCTCCACTTGACCAAGAAAAAAGTGATTC TCCCAGAAGCACAAAGCATACTCTTGCCCC TCAGGTGTTGCTTGTGTACATCGTACCCATCCATTCGGCTTCACCTGCAGCCAACGGCCTGGAATCGCAAAGAGACACCACTCTGGGCAGAGCAGAGCAGGGTAT GGGGTGGGGAGAGAGGGTGGAGGGTTTTATAAACAAACTTAACAGCAATATTGAAAGGAGGTGGGGGATTGAGGGAGGGACAGAGTGTTGGAGGGCCAGAGACTA GTCCTGAGATGGAAACAGCAACTTGTACAGTGGCTGAGAAAATAGGATATAGTTTTGATTTTTTTAATTGTAAAATATTTTGGAGGGAGAACAAAATCTTTTAAC ATTTTGAMTANATTTAGAGTTATATAAAAAAAAAAAAAA--
\end{abstract}

Figure 6. Nucleotide sequence of active RCCl cDNA clones, pcD40 and pcD51, and the encoded amino acid sequence of human $\mathrm{RCCl}$ cDNA. The complete nucleotide sequence of the pcD40 clone is shown in $A$. The sequence of pcD51 is shown in $B$, except for the putative coding region shared with the pcD40. Nucleotide sequences between two arrowheads are shared by both cDNAs.

Fig. 9|, showed a positive signal. To distinguish whether the $\mathrm{RCCl}$ gene is located on human chromosome 1 or 2 , chromosomes from the human lymphoblastoid cell line GM3876, which carries reciprocally translocated chromosomes $t(1 ; 20)$ was then sorted and examined. Among the six fractions $(a-f$, Fig. $9 B)$, fraction $b$, in addition to fraction a, hybridized to the fragment $\mathrm{C}$ probe. Fraction b contains two translocated chromosomes $(1 ; 20)$ (1qter1 p13 : : 20q133-20qter) and $(1 ; 20)(1 \mathrm{pl3}-1$ pter : : 20pter20q133), but not chromosome 2. From these results, we concluded that the human $\mathrm{RCCl}$ gene is located on chromosome 1 . 


\section{Amino acid number}

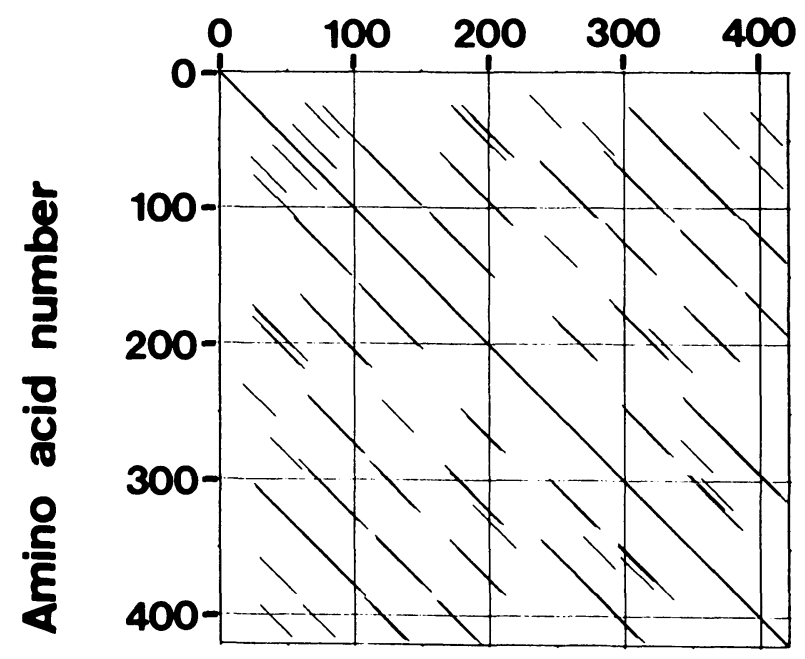

Figure 7. Comparison of the amino acid sequence predicted from the RCCl cDNA with itself. A computer program was used to generate the homology matrix, with a window size of 25 residues long.

\section{Discussion}

The $t s \mathrm{BN} 2$ cell line shows several temperature-sensitive phenotypes, such as the inhibition of $G_{1}$ progression $\left(G_{1}\right.$ type), the inhibition of $S$ progression ( $S$ type), and the induction of PCC (Nishimoto et al. 1978, 1981). The reversion rate of $t s \mathrm{BN} 2$ cells is $1.6 \times 10^{-8}$ (Nishimoto and Basilico 1978), which is close to the rate of spontaneous mutation in any single gene (Luria and Delbrück 1943), thereby suggesting that all temperature-sensitive phenomena of $t s \mathrm{BN} 2$ cells must be caused by a single mutation.

Previously we considered that $t s \mathrm{BN} 2$ cells had a primary defect in the DNA synthesis, so that $t s B N 2$ cells were one of the $\mathrm{DNA}^{-}$mutants. Since normal chromosome condensation occurs after the completion of the $S$ phase, the premature cessation of DNA synthesis at the nonpermissive temperature might provide a signal for PCC. But this argument seems to be unlikely because of the following:

(1) The inhibition of DNA synthesis alone cannot induce PCC. To induce PCC in normal cells, drugs such as caffeine are required in addition to inhibition of DNA synthesis (Schlegel and Pardee 1986). PCC induction by caffeine is very similar to the case of $t s \mathrm{BN} 2$ cells (Schlegel et al. 1987), thereby suggesting that the $t s B N 2$ mutation may have an effect similar to caffeine, rather than inhibiting DNA synthesis. (2) No residual DNA synthesis at the nonpermissive temperature is required for PCC induction of $t s B N 2$ cells, since PCC can be induced in $t s B N 2$ cells synchronized at the $G_{1} / S$ boundary by hydroxyurea, even in the presence of drugs that inhibit DNA synthesis (Nishimoto et al. 1981). (3) PCC occurred even in tsBN2 cells that are blocked in the $\mathrm{G}_{2}$ phase with neocarzinostatin, by shifting the temperature to the nonpermissive one (Ishida et al. 1985). (4) Inhibition of RNA and protein synthesis observed in the very early $G_{1}$ phase at the nonpermissive temperature (Nishimoto et al. 1981) cannot be caused by inhibition of DNA synthesis. Thus, the primary defect of $t s \mathrm{BN} 2$ cells does not seem to be the inhibition of DNA synthesis.

PCC is observed only in the $S$ and $G_{2}$ phases. Since chromosome condensation inhibits the synthesis of DNA, RNA, and protein, it is reasonable to assume that inhibition of the S-phase progression is due to PCC. Although, microscopically, PCC induction could not be observed in the $G_{1}$ phase, both RNA and protein synthesis are inhibited in this phase at the nonpermissive temperature $\left(39.5-40.5^{\circ} \mathrm{C}\right)$. The reduction of RNA and protein synthesis will inhibit the progression of the $G_{1}$ phase, but the actual cause of the inhibition of RNA and protein synthesis is not clear. We considered that inhibition of RNA and protein syntheses in the $G_{1}$ phase might be caused by premature chromosome condensation, because slight, but significant, increases in histone $\mathrm{H} 1$ phosphorylation were observed in the $G_{1}$ phase at the nonpermissive temperature (Ajiro et al. 1983). Furthermore, the occurrence of PCC in the $G_{1}$ phase was suggested by the following experiment: Postirradiation incubation $\left(3 \mathrm{hr}\right.$ at $\left.40^{\circ} \mathrm{C}\right)$ of $\mathrm{x}$-irradiated $t s \mathrm{BN} 2$ cells in-

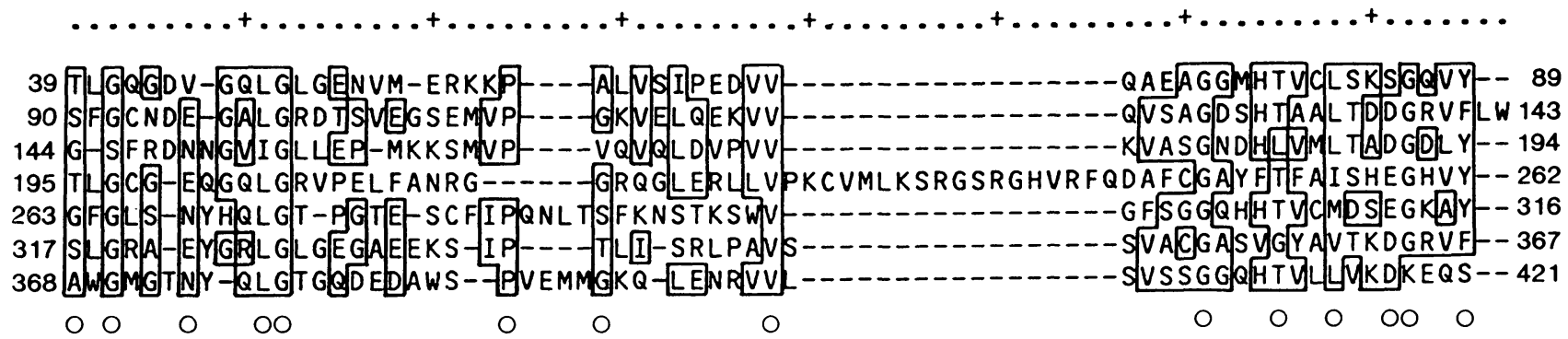

Figure 8. Alignment of the amino acid sequences among seven repeats. Gaps |- | are inserted to increase sequence similarity. Most common amino acids (including chemically similar amino acids) are boxed. Classification of amino acids based on their chemical similarity is as follows: A, T,G,P,S; D,E,N,Q; H,K,R; I,L,M,V; F, Y,W,$C$ (Schwartz and Dayhoff 1978). Highly conserved positions where six or seven repeats share identical or chemically similar amino acids are marked by open circles. The position numbers corresponding to the start and the end of each repeat are also shown. 


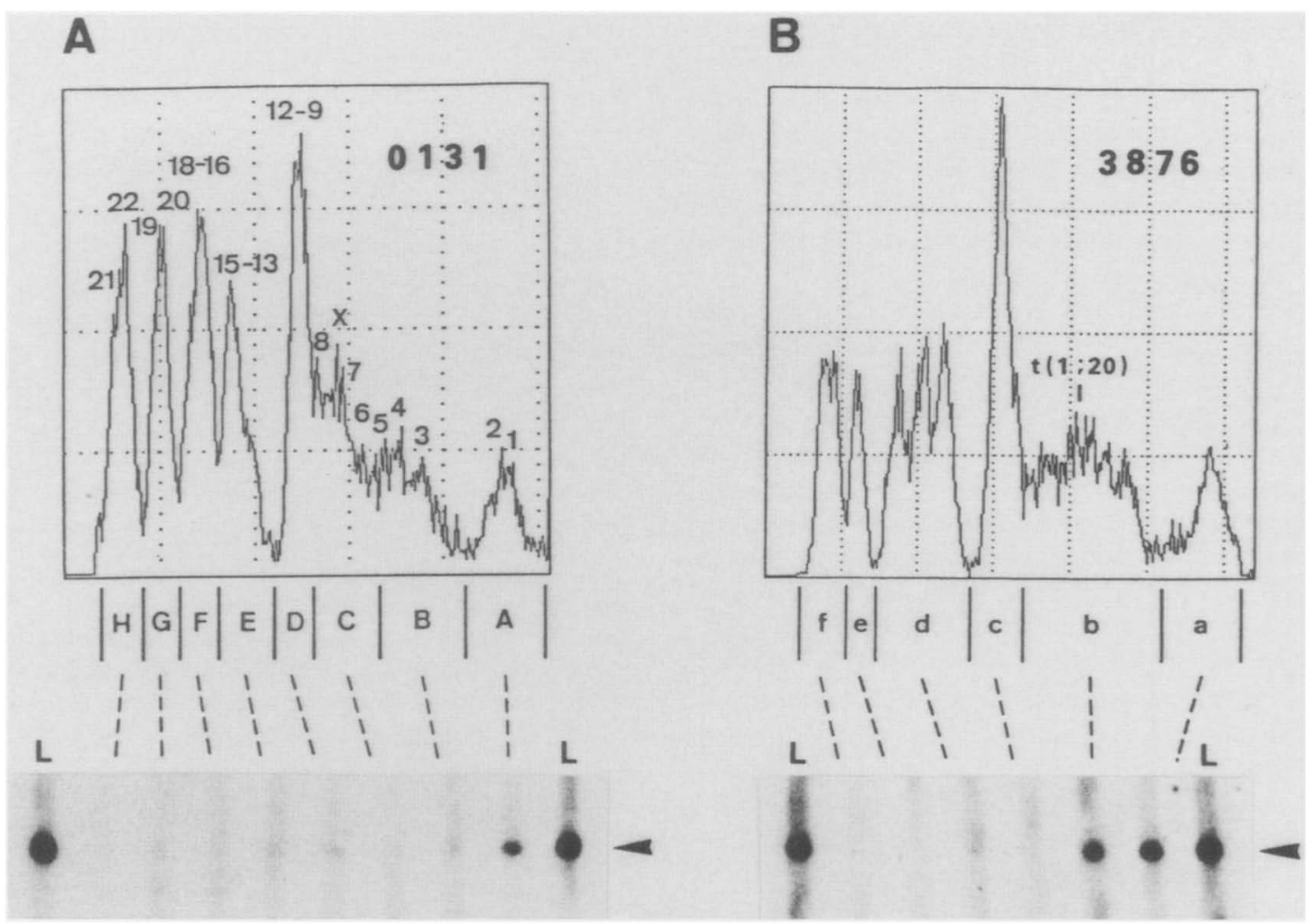

Figure 9. Assignment of human $\mathrm{RCCl}$ gene to chromosome 1. $(A)$ Flow histogram of normal human chromosomes from the cell line GM0131. Numbers in the figure represent the positions of human chromosomes. Chromosomes were stained with ethidium bromide and sorted into fractions $A-H$, as described by Fukushige et al. (1986). DNA was prepared from the sorted chromosomes, digested by PvuII, and subjected to Southern blot analysis (Southern 1975) using a ${ }^{32}$ P-labeled human RCCl probe C (shown in Fig. 1). (Lane $L$ ) Total human lymphocyte DNA. The arrow indicates the position of the DNA fragment hybridizing to the probe. $(B)$ Flow histogram of chromosomes from a human cell line GM 3876 that carries a reciprocal translocation $t(1 ; 20)$. DNAs were extracted from sorted chromosomes and subjected to Southern analysis as described in $A$.

creased the killing effect of $\mathrm{X}$ rays. This increment of killing effect was diminished by addition of cycloheximide (Sasaki and Nishimoto 1987), suggesting that $\mathrm{x}$-irradiated $t s \mathrm{BN} 2$ cells become more sensitive to $\mathrm{X}$ rays, depending on the new synthesis of protein(s) at the nonpermissive temperature. The effect of postirradiation incubation can be observed in both the $G_{1}$ and $S$ phases, suggesting that some protein(s) that induce PCC at the $S$ phase might be synthesized even in the $G_{1}$ phase at the nonpermissive temperature. Thus, we assumed that all the temperature-sensitive phenomena observed in $t s \mathrm{BN} 2$ cells might be caused by a single event-premature chromosome condensation. The finding that $t s \mathrm{BN} 2$ cells can be transformed to the ts ${ }^{+}$phenotype by a single cDNA is compatible with this proposal.

The isolated RCC1 cDNA transforms tsBN2 cells to the ts ${ }^{+}$phenotype with an efficiency comparable to that of the genomic RCCl cosmid DNAs. The RCCl cosmids are transcribed under control of the promoter of the RCCl gene, but the inserted $\mathrm{cDNAs}$ of $\mathrm{pcD}$ clones are transcribed under control of the SV40 promoter, which has no signal for the cell-cycle-specific transcription. Therefore, for regulation of the onset of chromosome condensation, the RCCl gene may not need to be transcribed at a precise time in the cell cycle.
The human $\mathrm{RCCl}$ gene was found to code for a $2.5-\mathrm{kb}$ poly $(\mathrm{A})^{+} \mathrm{RNA}$, both in HeLa and $t s \mathrm{BN} 2$ cells. Apparently, the RCCl gene is well conserved between hamsters and humans, thereby suggesting that this gene has a fundamental role in promoting growth.

Two active RCCl cDNAs (the base sequences at the 5 '-proximal region which differ) share a common open reading frame, encoding a protein composed of 421 amino acids. Since two active cDNAs have a comparable efficiency to complement the $t s \mathrm{BN} 2$ mutation, we concluded that this ORF encoded the $\mathrm{RCCl}$ product. Both cDNAs hybridized to the B95 cosmid DNA. However, the $5^{\prime}$ proximal region of cDNAs hybridized to a different fragment of the $5^{\prime}$ genomic DNA region. Thus, we considered that the two cDNAs were derived from the same human $\mathrm{RCCl}$ gene, but were transcribed from different promoters. The $3^{\prime}$ untranslated region of pcD40 does not have a common polyadenylation signal and is shorter than pcD51. However it is reasonable to assume that the original mRNA of the pcD40 has the same 3' untranslated region as the pcD5l clone, since the pcD40 does not have any extra different base sequences in this region, compared with that of pcD51. Thus, two mRNA species with a similar size $(2.5-2.6 \mathrm{~kb})$ are probably transcribed from the same human $\mathrm{RCCl}$ gene. 
The putative $\mathrm{RCCl}$ gene product has seven tandemly arranged homologous domains of about 60 amino acids. These repeated domains show a significant homology and the deduced amino acid composition is rich in glycine. Several proteins with repetitive homologous domains have been reported. No significant homology between such repetitive sequences and those of the human $\mathrm{RCCl}$ protein has been detected.

With regard to function of the RCCl product, we propose the following argument, assuming that the isolated human RCCl gene corresponds to the $t s$ mutated gene in the $t s \mathrm{BN} 2$ cell line. Our previous work suggested that the PCC induction in $t s B N 2$ cells is regulated in a posttranscriptional fashion (Nishimoto et al. 1981); thus, the $\mathrm{RCCl}$ product may repress translation of a mRNA encoding chromosome-condensing protein(s) from $G_{1}$ to the $S$ phase of the cell cycle. Sachs et al. (1986) found that the polyadenylate-binding protein has a region composed of four tandemly arranged 90 -amino-acid residues. This protein is processed into a repeated domain and other portions, and the repeated domain forms a ribonucleoprotein complex by binding to the poly(A) region of mRNA. Thus, while it is tempting to speculate that the $\mathrm{RCCl}$ product belongs to the group of poly(A)-binding proteins, no homology was found between this protein and the $\mathrm{RCCl}$ product.

Another possibility is that the $\mathrm{RCCl}$ product is a DNA-binding protein and regulates the production of chromosome-condensing protein at the transcriptional level. Since actinomycin D partially inhibits PCC induction (Nishimoto et al. 1981), this possibility has not been ruled out.

The third possibility is that the $\mathrm{RCCl}$ product forms a complex with the chromosome-condensing protein, and if such is the case, the homologous repeated domain may be required to make a complex with the chromosome-condensing protein, and the $\mathrm{RCCl}$ protein may be a kind of anti-MPF protein that is supposed to be involved in events linked to the progression from $M$ to the $G_{1}$ phase (Adlakha et al. 1983; Newport and Kirschner 1984).

The $t s$ mutated gene in $t s \mathrm{BN} 2$ cells seems to be involved in regulating the onset of chromosome condensation. Therefore, the isolated human RCCl gene, even if it is a kind of suppressor gene for the original mutation in $t s \mathrm{BN} 2$ cells, might be involved in the regulatory process for the onset of chromosome condensation. To determine whether the isolated human $\mathrm{RCCl}$ gene corresponds to the original mutated gene in the $t s \mathrm{BN} 2$ cell line, the hamster RCCl gene from BHK21 and tsBN2 cell lines is now being cloned.

\section{Materials and methods}

\section{Cell lines and media}

The $t s \mathrm{BN} 2-\mathrm{N} 9$ cell line is a thymidine kinase-negative derivative of the $t s \mathrm{BN} 2$ cell line, a temperature-sensitive mutant of the BHK21 cell line (Nishimoto and Basilico 1978). The ST2-7 cell line is a secondary ts ${ }^{+}$transformant of $t s B N 2-N 9$ cells (Kai et al. 1986). The HeLa cell line is a carcinoma cell line derived from the human uterine cervix. These cell lines were maintained in Dulbecco's modified Eagle medium supplemented with $10 \%$ calf serum, in a humidified atmosphere containing $10 \% \mathrm{CO}_{2}$.

Two human lymphoblastoid cell lines-GM0131, 46,XX, and GM3876, 46,XY, t(1:20)(1qter-1p13::20q133-20qter; 20pter-20q133: : 1p13-1pter)-were obtained from The Human Genetic Mutant Cell Repository (Camden, New Jersey). These lines were grown in RPMI 1640 medium supplemented with $10 \%$ fetal calf serum in humidified $5 \% \mathrm{CO}_{2} / 95 \%$ air. The $t s \mathrm{BN} 2-\mathrm{N} 9$ cell line was grown at $33.5^{\circ} \mathrm{C}$ and the ts ${ }^{+}$transformant cell line, ST2-7 was grown at $39.5^{\circ} \mathrm{C}$. Other cell lines were grown at $37^{\circ} \mathrm{C}$.

\section{Nucleic acid isolation and filter hybridization}

Cytoplasmic and total cellular RNA were prepared according to Maniatis et al. (1982). Polyadenylated RNAs were fractionated, using oligo(dT)-cellulose chromatography. Fractionated poly $(\mathrm{A})^{+}$RNAs were denatured in formaldehyde/formamide, electrophoresed in $1.5 \%$ agarose-formaldehyde gels, and transferred to nitrocellulose filters, according to Thomas (Thomas 1980). The nitrocellulose filters were hybridized under highly stringent conditions with ${ }^{32}$ P-labeled, nick-translated DNA probes.

\section{Isolation of cDNA clone}

A cDNA library made from SV40-transformed human fibroblasts was generously provided by Dr. Paul Berg (Okayama and Berg 1983) and screened using the gel-purified 1.5-kb EcoRIBamHI fragment (probe C) (Fig. 1) derived from cosmid B95.

\section{DNA transfection}

The $t s$ BN2-N9 cell line was plated at a concentration of $7.5 \times 10^{5}$ cells per $100-\mathrm{mm}$ dish and transfected with DNA extracted from cosmid B95 or RCCl cDNA clones. As a carrier, $20 \mu \mathrm{g}$ of DNA from $t s \mathrm{BN} 2$ cells was transfected simultaneously. After transfection, the cultures were incubated at $33.5^{\circ} \mathrm{C}$ for $36 \mathrm{hr}$ and then at $39.5^{\circ} \mathrm{C}$ for 9 days. The ts ${ }^{+}$colonies were stained and counted.

\section{DNA sequencing}

cDNA inserts of pcD40 and pcD51 were digested with appropriate restriction endonucleases, and the resulting fragments were subcloned into M13-based cloning vectors, according to Barnes et al. (1983). The nucleotide sequence was determined by the dideoxy sequencing method developed by Sanger et al. (1977).

\section{Dot matrix analysis}

Computer-assisted comparison of amino acid sequences was performed according to Toh et al. (1983).

\section{Chromosomal assignment}

Chromosomal assignment was performed using the method of chromosome sorting combined with Southern hybridization (Southern 1975) as described by Fukushige et al. (1986). 


\section{Acknowledgments}

This study was supported in part by grants from the Ministry of Education, Science and Culture, and by a grant from the Ministry of Health and Welfare, Japan. We thank Dr. Teruo Yasunaga (The Institute of Physical and Chemical Research) for assistance with computer analysis of the base sequence, Dr. Paul Berg for kindly sending us the human cDNA library, and $\mathrm{M}$. Ohara for comments on the manuscript.

\section{References}

Adlakha, R.C., C. Sahasrabuddhe, D.A. Wright, H. Sahasrabuddhe, H. Bigo, and P.N. Rao. 1983. Evidence for the presence of inhibitors of mitotic factors during Gl period in mammalian cells. J. Cell Biol. 97: 1707-1713.

Ajiro, K., T. Nishimoto, and T. Takahasi. 1983. Histone $\mathrm{Hl}$ and H3 phosphorylation during premature chromosome condensation in a temperature-sensitive mutant (tsBN2) of baby hamster kidney cells. I. Biol. Chem. 258: 4534-4538.

Barnes, W.M., M. Bevan, and P.H. Son. 1983. Kilo-sequencing: Creation of an ordered nest of asymmetric deletions across a large target sequence carried on phage M13. Methods Enzymol. 101: 98-122.

Berget, S.M. 1984. Are U4 small nuclear ribonucleoproteins involved in polyadenylation? Nature 309: 179-182.

Fukushige, S., T. Murotsu, and K. Matsubara. 1986. Chromosomal assignment of human genes for gastrin, thyrotropin (TSH)-subunit and c-erb B-2 by chromosome sorting combined with velocity sedimentation and Southern hybridization. Biochem. Biophys. Res. Commun. 134: 477-483.

Hayashi, A., S. Yamamoto, T. Nishimoto, and T. Takahashi. 1982. Chromosome condensing factor(s) induced in tsBN2 cells at a nonpermissive temperature: Evidence for transferable material by cell fusion. Cell. Struct. Funct. 7: 291-294.

Ishida, R., T. Takahashi, and T. Nishimoto. 1985. Chromosomes of G2 arrested cells are easily analyzed by use of the tsBN2 mutation. Cell Struct. Funct. 10: 417-420.

Johnson, R.T. and P.N. Rao. 1970. Mammalian cell fusion: Induction of premature chromosome condensation in interphase nuclei. Nature 226: 717-722.

Kai, R., M. Ohtsubo, M. Sekiguchi, and T. Nishimoto. 1986. Molecular cloning of a human gene that regulates chromosome condensation and is essential for cell proliferation. Mol. Cell. Biol. 6: 2027-2032.

Kozak, M. 1984. Compilation and analysis of sequences upstream from the translational start site in eukaryotic mRNAs. Nucleic Acids Res. 12: 857-872.

Luria, S. and M. Delbrück. 1943. Mutations of bacteria from virus sensitivity to virus resistance. Genetics 28: 491-511.

Maniatis, T., E.F. Fritsch, and J. Sambrook. 1982. Molecular cloning: A laboratory manual. Cold Spring Harbor Laboratory, Cold Spring Harbor, New York.

Marcel van Duin, J. Wit, H. Odijk, A. Westerveld, A. Yasui, M.H.M. Koken, J.H.J. Hoeijmakers, and D. Bootsma. 1986. Molecular characterization of the human excision repair gene ERCC-1: cDNA cloning and amino acid homology with the yeast DNA repair gene RAD 10. Cell 44: 913-923.

Newport, J. and M.W. Kirschner. 1984. Regulation of the cell cycle during early Xenopus development. Cell 37: 731-742.

Nishimoto, T. and C. Basilico. 1978. Analysis of a method for selecting temperature sensitive mutants of BHK cells. Somat. Cell Genet. 4: 323-340.

Nishimoto, T., E. Eilen, and C. Basilico. 1978. Premature chromosome condensation in a tsDNA ${ }^{-}$mutant of BHK cells. Cell 15: 475-483.
Nishimoto, T., R. Ishida, K. Ajiro, S. Yamamoto, and T. Takahashi. 1981. The synthesis of protein(s) for chromosome condensation may be regulated by a post-transcriptional mechanism. J. Cell. Physiol. 109: 299-308.

Okayama, H. and P. Berg. 1983. A cDNA cloning vector that permits expression of cDNA inserts in mammalian cells. Mol. Cell. Biol. 3: 280-289.

Prescott, D.M. 1976. The cell cycle and the control of cellular reproduction. Adv. Genet. 18: 99-177.

Proudfoot, N.J. and G.G. Brownlee. 1976. 3' Non-coding region sequences in eukaryotic messenger RNA. Nature 263: $211-$ 214.

Rao, P.N., B. Wilson, and T.T. Puck. 1976. Premature chromosome condensation and cell cycle analysis. I. Cell. Physiol. 91: $131-142$.

Sachs, A.B., M.W. Bond, and R.D. Kornberg. 1986. A single gene from yeast for both nuclear and cytoplasmic polyadenylatebinding proteins: Domain structure and expression. Cell 45: $827-835$.

Sanger, F., S. Nicklen, and A.R. Coulson. 1977. DNA sequencing with chain terminating inhibitors. Proc. Natl. Acad. Sci. 74: 5463-5467.

Sasaki, H. and T. Nishimoto. 1987. Chromosome condensation may enhance X-ray-related cell lethality in a temperaturesensitive mutant (tsBN2) of BHK21 cell. Radiat. Res. 109: 407-418.

Schlegel, R. and A.B. Pardee. 1986. Caffeine-induced uncoupling of mitosis from the completion of DNA replication in mammalian cells. Science 232: 1264-1266.

Schlegel, R., R.G. Croy, and A.B. Pardee. 1987. Exposure to caffeine and suppression of DNA replication combine to stabilize the proteins and RNA required for premature mitotic events. I. Cell. Physiol. 131: 85-91.

Schwartz, R.M. and M.O. Dayhoff. 1978. Atlas of protein sequence and structure, vol. 5, suppl. 3 (ed. M.O. Dayhoff), pp. 353-358. National Biomedical Research Foundation, Washington, DC.

Southern, E. 1975. Detection of specific sequences among DNA fragments separated by gel electrophoresis. I. Mol. Biol. 98: $503-517$.

Sunkara, P.S., D.A. Wright, and P.N. Rao. 1979. Mitotic factors from mammalian cells induce germinal vesicle breakdown and chromosomal condensation in amphibian oocytes. Proc. Natl. Acad. Sci. 76: 2799-2802.

Thomas, P.S. 1980. Hybridization of denatured RNA and small DNA fragments transferred to nitrocellulose. Proc. Natl. Acad. Sci. 77: 5201-5205.

Toh, H., H. Hayashida, and T. Miyata. 1983. Sequence homology between retroviral reverse transcriptase and putative polymerases of hepatitis B virus and cauliflower mosaic virus. Nature 305: 827-829.

Yamashita, K., F.M. Davis, P.N. Rao, M. Sekiguchi, and T. Nishimoto. 1985. Phosphorylation of nonhistone proteins during premature chromosomal condensation in a temperature-sensitive mutant, tsBN2. Cell. Struct. Funct. 10: 259270 . 


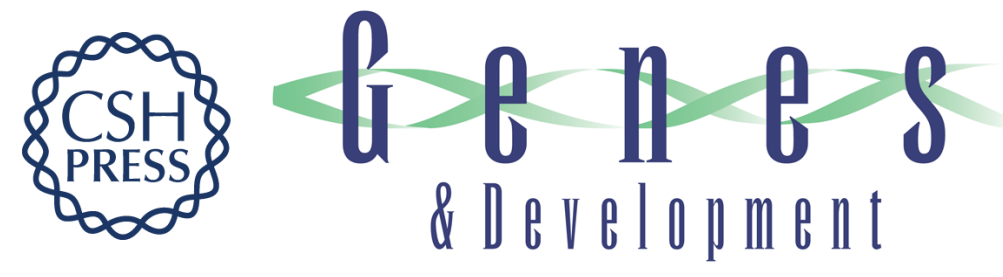

\section{Isolation and characterization of the active cDNA of the human cell cycle gene (RCC1) involved in the regulation of onset of chromosome condensation.}

M Ohtsubo, R Kai, N Furuno, et al.

Genes Dev. 1987, 1:

Access the most recent version at doi:10.1101/gad.1.6.585

References This article cites 30 articles, 9 of which can be accessed free at:

http://genesdev.cshlp.org/content/1/6/585.full.html\#ref-list-1

License

Email Alerting
Service

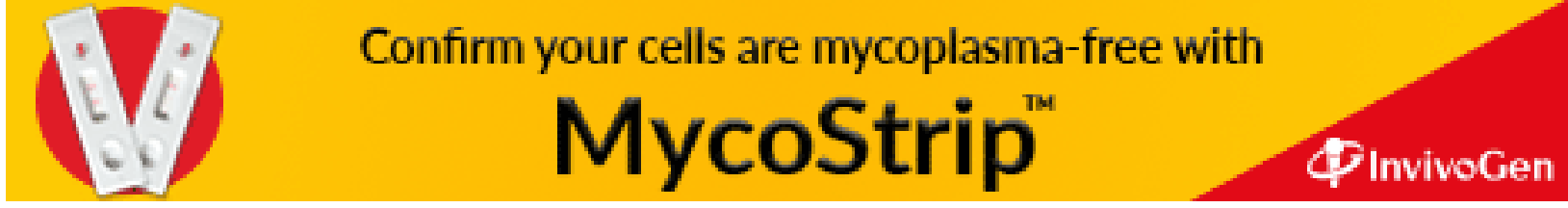

